\title{
A PASSIVE-TELEMETRY-BASED PRESSURE SENSING SYSTEM
}

\author{
Andrew DeHennis and Kensall D. Wise
}

\author{
NSF Engineering Research Center for Wireless Integrated MicroSystems \\ Department of Electrical Engineering and Computer Science \\ The University of Michigan, Ann Arbor, MI 48109-2122
}

\begin{abstract}
This paper presents a wireless battery-free pressure sensing system. The system utilizes a bulk micromachined capacitive pressure sensor along with a capacitive interface circuit implementing passive absorption telemetry. The pressure transducer is based on the dissolved wafer process and has been designed to test the scalability of polysilicon-to-glass anodic bonding. The sensors have been fabricated realizing a $100 \mu \mathrm{m}$-wide bonding ring sealed at wafer level. The sensor interface implements a capacitance-tofrequency converter with a sensitivity of $377 \mathrm{~Hz} / \mathrm{pF}$ for readout of the capacitive pressure sensor and reference capacitors. The wireless system utilizes inductively coupled power and frequency-encoded backscatter modulation for transmission of sensor readout data.
\end{abstract}

\section{INTRODUCTION}

Wireless data gathering systems have the potential to restructure the instrumentation used in a variety of industries, including security, health care, and transportation. In looking at the possibilities for the implementation of telemetry, we find two methods: active telemetry and passive telemetry. Active telemetry provides two-way communication over a long range [1]; however, systems usually consume a relatively large volume and have a finite lifetime due to battery limitations. Passive telemetry reduces the transmission distance; however, it does allow the implementation of battery-free wireless devices. There are currently two methods of implementing passive telemetry systems. One method uses a fully passive sensing device that shifts the resonant frequency of an LC tank and can be monitored through a wireless link [2]. Implementing circuitry with the wireless device, passive telemetry can modulate a reflected load to the remote powering transceiver [3,4]. Radio Frequency Identification (RFID) devices have commercialized passive telemetry systems for the readout of wireless, battery-free tags for applications in tracking and security, among others [5]. Extending this technology in its integration with sensors and actuators then begins to allow a whole range of applications. MEMS technology is laying the groundwork for the practical realization of such devices in its development of fabrication processes that allow for full integration of the system at the wafer level [6]. Wireless passive telemetry systems based on the integration of MEMS and RF backscatter modulation have been realized for neural recording [7] and intraocular pressure monitoring [8]. A passive wireless system that provides sensing capability needs not only the implementation of passive telemetry circuitry but also the realization of both the sensor interface circuitry and the sensors themselves. Current work on the integration of passive telemetry with sensing systems aims to define the size/range/power tradeoffs for such devices.

\section{THEORETICAL ANALYSIS}

The two essential parts of passive telemetry systems are the ability to couple power to the wireless system and the ability to recover the modulated backscattered signal. Previous work has presented the theory governing the power transmission through inductive coupling $[2,5,7,9]$. This investigation has focused on an analysis of the modulated backscattering transmission technique, looking at an inductively coupled parallel LCR tank (representing the wireless monitoring system) and the remote powering and datareceiving antenna [3]. The equivalent circuit model for this system is shown in Fig. 1. The modulated backscattering technique is

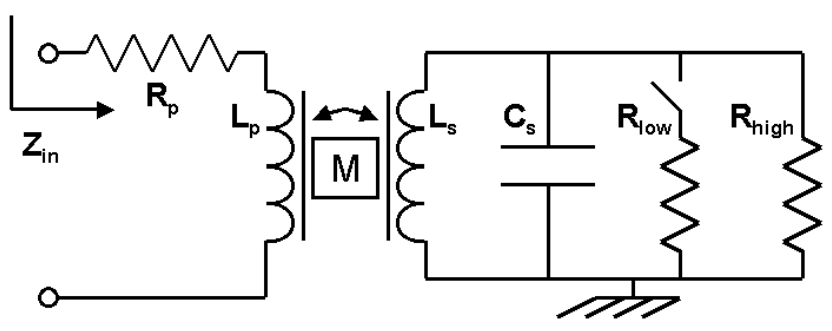

Fig. 1: Equivalent circuit model for passive absorption telemetry, generating a modulated load for the primary antenna.

implemented by switching between an equivalent high-Q LCR tank and an equivalent low-Q LCR tank. Analysis of the input impedance of the coupled primary inductor at the resonant frequency of the secondary LC tank reveals

$$
Z_{\text {in }}\left(\omega_{0}\right)=R_{P}+\omega_{0} L_{P}\left(i-i k^{2}+k^{2} Q_{\text {tank }}\right)
$$

where $R_{p}$ and $L_{p}$ are the respective resistance and inductance of the primary antenna, $k$ is the coupling coefficient between $L_{P}$ and $L_{S}$ [2,5], and $Q_{\text {tank }}$ is the quality factor of the parallel LCR tank defined at resonance by

$$
Q_{\text {tank }}=R_{\text {load }} \sqrt{\frac{C_{S}}{L_{S}}}
$$

where $R_{\text {load }}$ represents the load for the coupled wireless system defined by $R_{\text {high }}$ when the loading switch is open and $R_{\text {low }}$ when the loading switch is closed. The backscattered signal can be found by the difference between the two states such that

$$
\Delta Z_{\text {in }}\left(\omega_{0}\right)=\left|Z_{\text {in }}\left(\omega_{0}, Q_{\text {high }}\right)\right|-\left|Z_{\text {in }}\left(\omega_{0}, Q_{\text {low }}\right)\right|
$$

where $Q_{\text {high }}$ and $Q_{\text {low }}$ are the quality factors $\left(Q_{\text {tank }}\right)$ of the loaded and unloaded parallel LCR tanks, respectively. When applied to a passive telemetry system, the primary inductor is also responsible for inductively coupling power to the wireless system. Thus, a high-powered AC signal is generated into $L_{p}$ through the external circuitry $[7,9]$. The modulated loading of the parallel LCR tank reflects an amplitude modulation (AM) on the carrier. The received signal can be defined by the percent modulation of the carrier by 


$$
\frac{\Delta V_{A M}}{V_{\text {carrier }}} \cong \frac{\Delta Z_{\text {in }}\left(\omega_{0}\right)}{\left|Z_{\text {in }}\left(\omega_{0}, Q_{\text {high }}\right)\right|}
$$

where $V_{A M}$ is the modulation voltage and $V_{\text {carrier }}$ is the amplitude voltage of the carrier waveform, thus defining the received AM signal at the readout antenna for modulated backscattered data transmission. Eq. (4) realizes the signal received by the remote powering antenna is a function of the carrier frequency defined by the resonance of the wireless device and the external transmitter, the coupling coefficient, $k$, the passive loading resistance, $R_{\text {low }}$, and the power consumption of the circuit represented by $R_{h i g h}$ and defining $Q_{\text {high }}$.

\section{SYSTEM DESIGN}

The system-level implementation of this wireless device has been designed to simplify the on-chip functionality needed, providing only readout and transmission of the sensor and reference information. This approach minimizes both the power consumption and the die area needed to implement the interface circuit for the wireless system. A block diagram for the passive telemetry system is shown in Fig. 2. The pressure

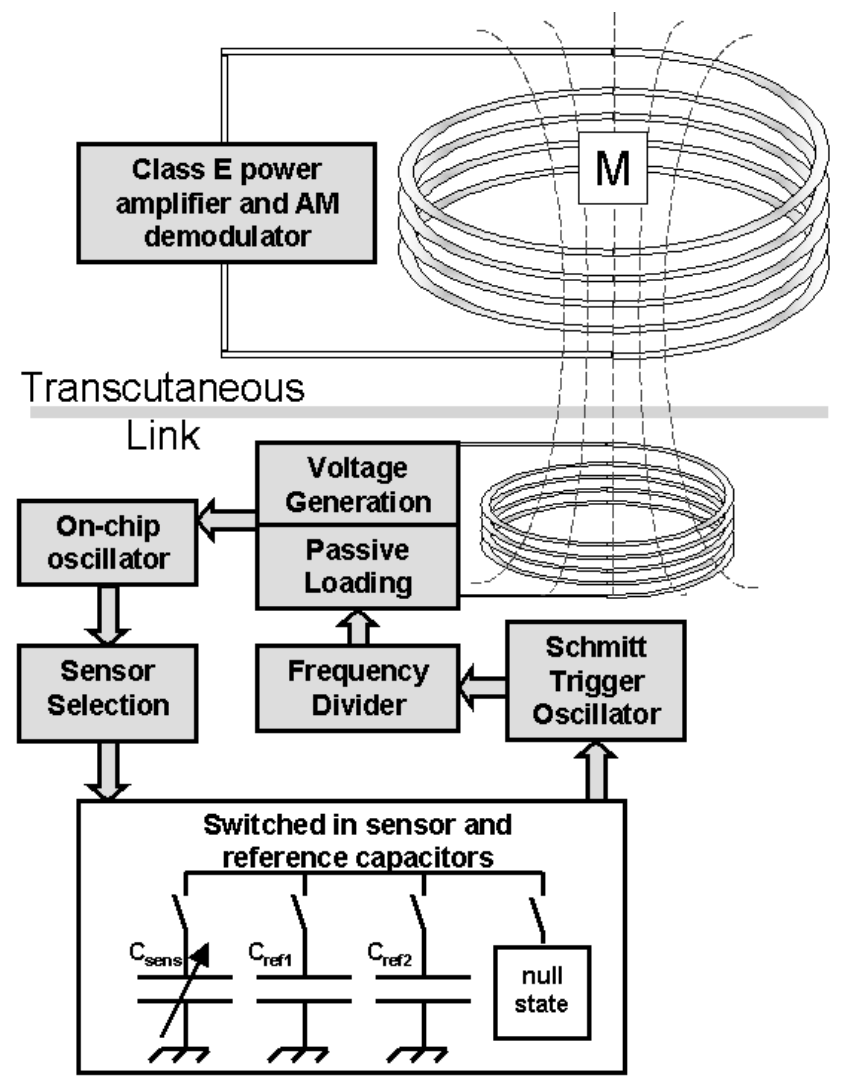

Fig. 2: Block diagram of the wireless pressure sensing system sensors used in this system are capacitive transducers. The capacitive signal from the transducer is digitized by the use of a capacitance-to-frequency (C-F) converter, which is implemented with a Schmitt-input ring oscillator with complimentary pass gates to provide the switching between the sensor, the reference capacitors, and the null state. The switchable $\mathrm{C}-\mathrm{F}$ conversion and frequency-division circuitry is shown in Fig. 3. The oscillation frequency of the C-F converter can be found by [10]

$$
f_{\text {osc }}=\frac{I_{o}}{2 V_{h} C_{s e n s}}
$$

where $V_{h}$ is the hysteresis of the Schmitt input stage, $C_{\text {sens }}$ is the sensor capacitance, and $I_{o}$ is the charging current determined by the output impedance of the drive transistors and the regulated power supply voltage. In passive telemetry applications, the regulated power supply can suffer from a high level of ripple due to rectified carrier feedthrough [3]. The implementation of the

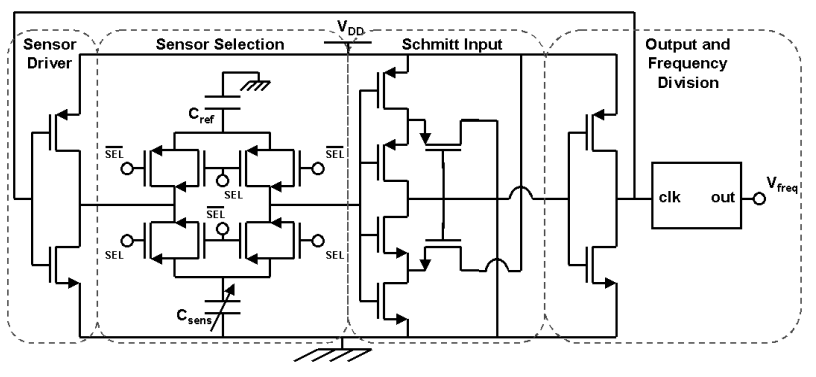

Fig. 3: A schematic of the switchable Schmitt trigger oscillator used for capacitance to frequency conversion.

Schmitt trigger oscillator provides a sensor interface that will not suffer from $V_{d d}$ ripple sensitivity as long as the ripple is kept below the $\mathrm{V}_{\mathrm{h}}$ hysteresis of the Schmitt input. The output of the oscillator is then used as the input to a frequency divider that generates a $50 \%$ duty-cycle digital square wave.

There is a second on-chip oscillator, which is fed into a frequency divider and a 4-bit decoder. The decoded signal is used to select the C-F readout to read one of four states: sensor readout, reference high, reference low, and a null state [11]. These four states are essential for wireless communication with the system. Reading of the two reference capacitors is necessary in order to compensate the system for temperature drift [12] and fluctuations in the availability of power for the wireless device [8,11]. Since switching between these states is done through an on chip oscillator, the null state is used to synchronize the coupled systems. The digital frequency-encoded information for the sensor and reference capacitors can then be sent to the RF front end of the system for transmission via absorption modulation. For batteryfree operation, a remote power source must be nearby in order to generate the RF carrier. The wireless system includes a resonant LC tank at the front end followed by a full-bridge rectifier. There is an added diode to protect the regulated signal from any power fluctuations caused by the actively loaded LC tank. The RF front end of the system is shown in Fig 4. The absorption modulation is done using the digital frequency output of the C-F converter to actively load the LC tank of the wireless system in controlling the switching of the shunting transistor $\mathrm{M}_{\mathrm{load}}$, which is the absorption modulation loading transistor. When the $\mathrm{M}_{\text {load }}$ transistor is open, the system couples rectified power through the blocking diode,

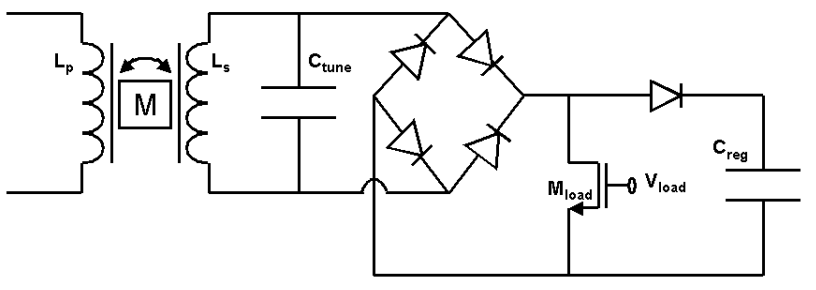

Fig. 4: Schematic of the $R F$ front end showing the rectification and load modulation implementation

charging up $\mathrm{C}_{\text {reg, }}$, which is the capacitor that provides power to all of the on-chip circuitry through the entire AC cycle and through 


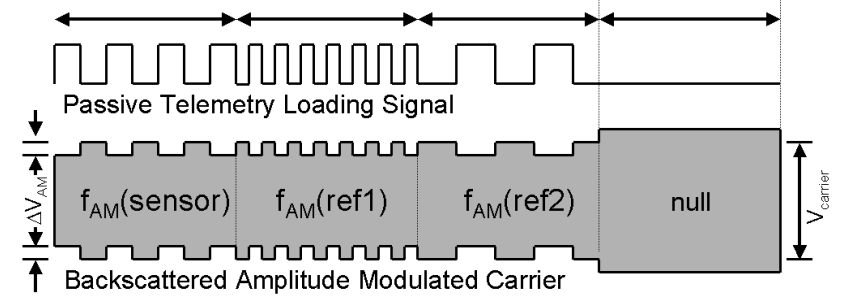

Fig. 5: AM frequency-encoded waveforms for the sensor readout of the wireless microsystem

the entire loading state. When the transistor is in the on state, the LC tank of the wireless system is shunted, which then generates a reflected load on the remote powering antenna. The transmission of the sensor and reference data is encoded in the amplitude modulation frequency. A visualization of the transmission protocol for the modulated backscattered signal is shown in Fig. 5. The system uses a Class E power amplifier [7,9] to generate the RF remote carrier with an added AM demodulator for decoding of the reflected loading signal [5]. The bandpass demodulator is set to filter out the high frequency carrier and the DC offset of the resonant power amplifier. The signal is then digitized for further filtering and digital signal processing. An initial hardware filter is needed due to the relatively large voltage levels on the resonant node of the transmitter.

(a)
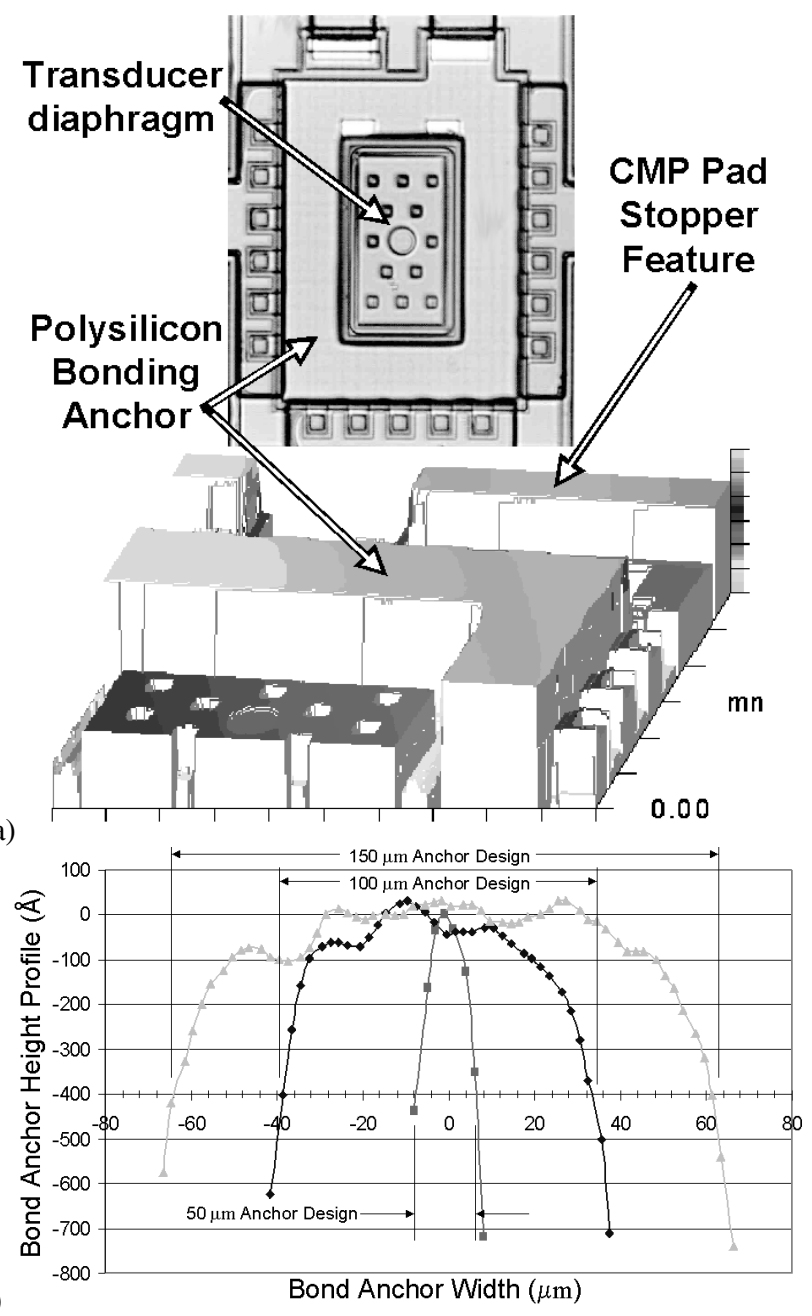

Fig. 6: (a) Silicon side of the capacitive pressure sensor before anodic bonding, (b) the effects of CMP on three different size anchor widths

\section{TRANSDUCER DESIGN AND FABRICATION}

The pressure transducers were fabricated using an anodically bonded silicon-on-glass process. The structure utilizes a two-layer polysilicon process to form hermetic feedthroughs to a sealed cavity $[6,12]$ and a $4 \mu \mathrm{m}$-thick boron-doped diaphragm. A polysiliconbonding ring is utilized to seal the cavity during the anodic bonding process. Chemical Mechanical Polishing (CMP) is used to decrease the surface roughness of the polysilicon to below $500 \AA$, which is needed to provide a uniform bond [12,13]. The CMP process has width scaling limitations due to the rounding of edges caused when polishing structures that have high topology, such as the recessedcavity pressure sensors. The transducers were designed to allow an investigation of the experimental limitations in scaling the bonding anchor width using this process. Pad stopper features were used to minimize the rounding effect that the CMP process had on the bonding ring [14]. The pre-bonded capacitive pressure transducer is shown along side an optical plot of the anchor areas and pad stopper features in Fig. 6(a). The pressure transducer has been fabricated with a surface micromachined plate inside the cavity to increase the sensitivity per unit area of the diaphragm [6]. A contour map was developed using optical spectroscopy across the polysilicon anchor areas before deposition of low temperature oxide [12], from which the CMP rounding effect is shown in Fig. 6(b). Anodic bonding tolerates a step height of no more than 50nm [13], which decreases the effective bonding width of the three anchor designs. The $150 \mu \mathrm{m}$, $100 \mu \mathrm{m}$ and $50 \mu \mathrm{m}$ anchor widths decreased to approximately $125 \mu \mathrm{m}$, $70 \mu \mathrm{m}$, and $15 \mu \mathrm{m}$, respectively, due to CMP. Uniform bonding rings around the anchors were only realized with the $100 \mu \mathrm{m}$ and $150 \mu \mathrm{m}$ designs. A uniform bond ring around the cavity was not achieved with the $50 \mu \mathrm{m}$ design, most likely due to the high amount of rounding on that design. The final design dimensions of the transducer diaphragm are $300 \mu \mathrm{m} \times 500 \mu \mathrm{m}$. This design achieved a $0.5 \mathrm{~mm} \times 0.75 \mathrm{~mm} \times 0.5 \mathrm{~mm}$ pressure sensor, sealed at wafer level. The sensor has a sensitivity of $0.2 \mathrm{fF} / \mathrm{mmHg}$, operating over a pressure range from 600 to $1000 \mathrm{mmHg}$.

\section{RESULTS}

The passive telemetry interface circuit was fabricated using a $3 \mu \mathrm{m}$ BiCMOS process (Fig. 7). On-chip poly-to-poly power

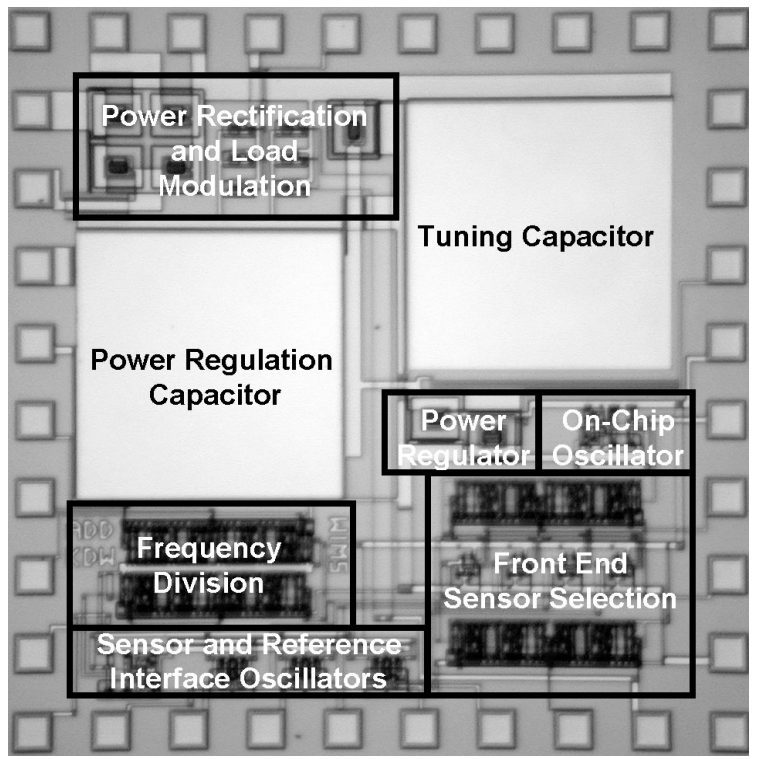

Fig. 7: Photograph the $2 m m x 2 m m$ passive-telemetry capacitive interface circuit realized using a $3 \mu \mathrm{m}$ 2P/1M BiCMOS process 


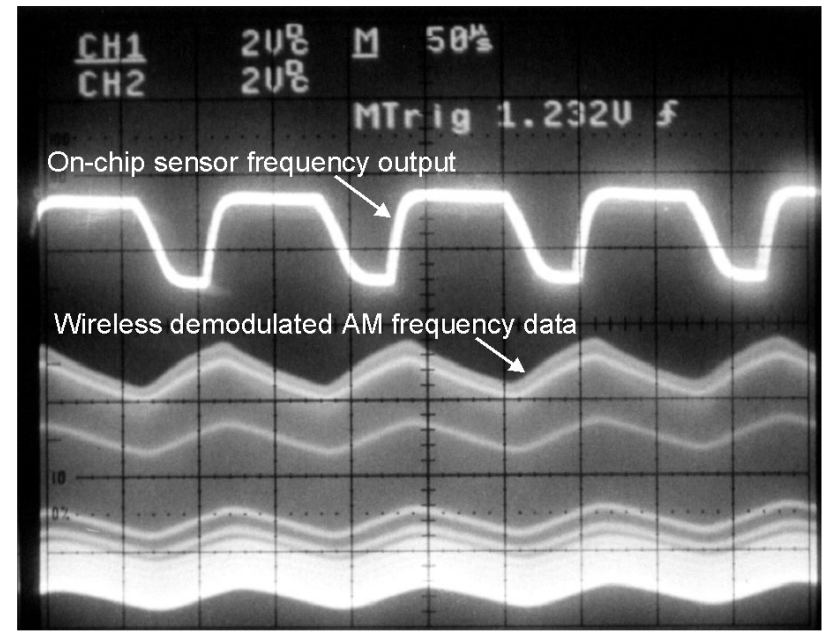

Fig. 8: Wireless system testing showing internal frequency output of the wireless system as well as the wirelessly received, first-order demodulated reverse telemetry signal.

regulation capacitors have been fabricated on-chip to allow for continuous functionality of the interface circuitry though the loaded and unloaded states of the system. Results from wireless testing using a Class $\mathrm{E}$ amplifier running at $800 \mathrm{kHz}$ are shown in Fig. 8. Testing has shown the power consumption of the interface circuit to be $1 \mathrm{~mW}$ running at $3 \mathrm{~V}$, which sets $R_{\text {high }}=18 \mathrm{k} \Omega$ for the high-Q state of the passive telemetry. The measured low-Q state for this system finds $R_{\text {low }}=433 \Omega$. An analysis of the percent modulation of this system predicted by Eq. (4) for a configuration with coupling coefficients scaling from $k=0.05$ to $k=0.025$ results in $17 \%$ to $2 \%$ amplitude modulation of the carrier, respectively. Characterization of the C-F converter (Fig. 9) shows a pressure sensitivity of $377 \mathrm{~Hz} / \mathrm{pF}$. At close coupling distances, the dominant noise source in the received wireless data is carrier feedthough, which sets the frequency resolution to approximately $30 \mathrm{~Hz}$ per cycle, without averaging. A higher performance version of this system can be configured using devices designed for higher sensitivity and lower dynamic range [2,12], obtaining a resolution less than $3 \mathrm{mmHg}$.

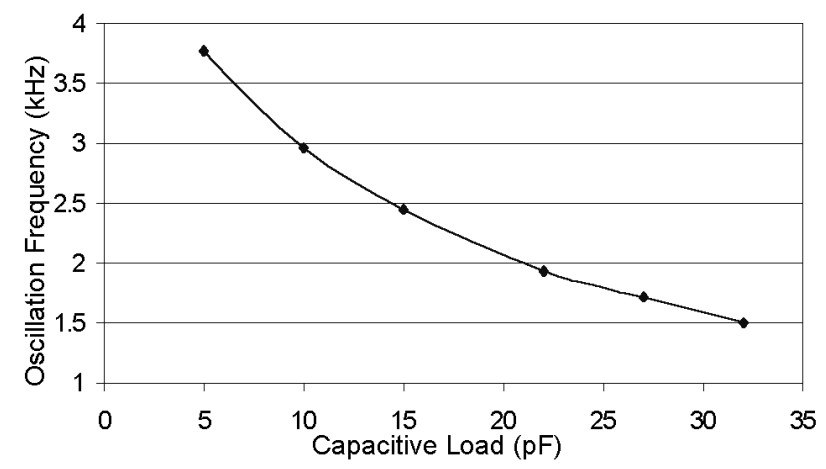

Fig. 9: Capacitance-to-frequency conversion characteristic of the interface chip with a $3 \mathrm{~V}$ regulated supply.

\section{CONCLUSIONS}

The design and realization of a battery-free pressure sensing system that utilizes remote inductive powering and passive telemetry has been presented. The theory governing the design of backscattered modulation telemetry devices has been developed. Capacitive pressure transducers have been fabricated realizing
$100 \mu \mathrm{m}$-wide polysilicon-to-glass bonding anchors sealed at wafer level. A passive telemetry interface that provides wireless frequency-encoded sensor readout has been implemented using a capacitance-to-frequency readout from the sensor. The realized circuit has a sensitivity of $377 \mathrm{~Hz} / \mathrm{pF}$ and can wirelessly resolve a capacitance change of less than $80 \mathrm{fF}$ at the transducer.

\section{ACKNOWLEDGMENTS}

This work is supported by the Engineering Research Centers Program of the National Science Foundation under Award Number EEC-9986866 and by a gift from Ms. Polly Anderson. The authors would like to thank Katharine Beach for her fabrication of the circuit.

\section{REFERENCES}

[1] A. M. Leung, et. al, "Intracranial Pressure Telemetry System Using Semicustom Integrated Circuits," IEEE Transactions on Biomedical Engineering, vol. BME-33, April 1986, pp. 386-395.

[2] A. DeHennis and K. D. Wise, "A Double-Sided Single-Chip Wireless Pressure Sensor," IEEE Conference on MicroElectroMechanical Systems, Las Vegas, NV, Jan. 2002, pp. 252-255.

[3] Q. Huang and M. Oberle, "A $0.5 \mathrm{~mW}$ Passive Telemetry IC for Biomedical Applications," IEEE Journal of Solid State Circuits, Vol. 33, No. 7, July 1998, pp. 937-946.

[4] Stavros Charzandroulis et al, "A Miniature Pressure System with a Capacitive Sensor and a Passive Telemetry Link for Use in Implantable Applications," Journal of MicroElectroMechanical Systems, Vol. 9, No. 1, March 2000, pp. 18-23.

[5] K. Finkenzeller, RFID Handbook, John Wiley \& Sons Ltd., Chichester, England, 1999.

[6] A. V. Chavan and K. D. Wise, "A Monolithic Fully-Integrated Vacuum-Sealed CMOS Pressure Sensor," IEEE Conference on Micro-ElectroMechanical Systems, Jan 2000, pp. 341-346.

[7] T. Akin, K. Najafi, and R. M. Bradley, "A Wireless Implantable Multichannel Digital Neural Recording System for a Micromachined Sieve Electrode," IEEE Journal of Solid State Circuits, Vol. 33, No. 1, January 1998, pp. 937-946.

[8] K. Stangel, et al, "A Programmable Intaocular CMOS Pressure Sensor System Implant," IEEE Journal of Solid-State Circuits, Vol. 36, No.7, July 2001, pp. 1094-1100.

[9] P. Troyk and M. Schwan. "Class E Driver for Transcutaneous Power and Data Link for Implanted Electronics Devices," Medical and Biological Engineering \& Computing, January 1992, pp. 69-75.

[10] Y. Matsumoto and M. Esashi, "Integrated Silicon Capacitive Accelerometer with PLL Servo Technique," Sensors and Actuators A, Vol. 39, 1993, pp 209-217

[11] J. Ji, S. Cho, Y. Zhang, K. Najafi, K. Wise, “ An Ultraminiature CMOS Pressure Sensor for a Multiplexed Cardiovascular Catheter," IEEE Transactions on Electron Devices, Vol 39, No 10, Oct. 1992, pp. 2260-2267.

[12] A. V. Chavan and K. D. Wise, "A Multi-Lead Vacuum-Sealed Capacitive Pressure Sensor," Digest Solid-State Sensor and Actuator Workshop, Hilton Head, June 1998, pp. 212-215

[13] S. Mack, H. Baumann, U. Gosele, "Gas Tightness of Cavities Sealed by Silicon Wafer Bonding," IEEE Conference on MicroElectroMechanical Systems, Nagoya, Japan, pp. 488-493, Jan. 1997.

[14] A. Chavan, An Integrated High Resolution Barometric Pressure Sensing System, Doctoral Thesis, University of Michigan, 2000 